\title{
Corrigendum
}

\section{Corrigendum to "Sensory-motor control in the ipsilesional upper extremity after stroke" [NeuroRehabilitation 9 (1997) 57-69]}

\author{
Patricia S. Pohl ${ }^{\mathrm{a}, *}$, Carolee J. Winstein ${ }^{\mathrm{b}}$, Somporn Onla-or ${ }^{\mathrm{b}}$ \\ ${ }^{a}$ Department of Physical Therapy Education and Center on Aging, University of Kansas Medical Center, 3901 Rainbow \\ Boulevard, Kansas City, KS 66160-7601, USA \\ ${ }^{\mathrm{b}}$ Department of Biokinesiology and Physical Therapy, University of Southern California, Los Angeles, CA, USA
}

The Publisher regrets that a table was inadvertently omitted from the above paper. The table should read as on following pages:

\footnotetext{
"PII of original article: S1053-8135(96)00014-0 
Table 1

Summary of studies on sensory-motor control in the ipsilesional upper extremity after unilateral brain damages

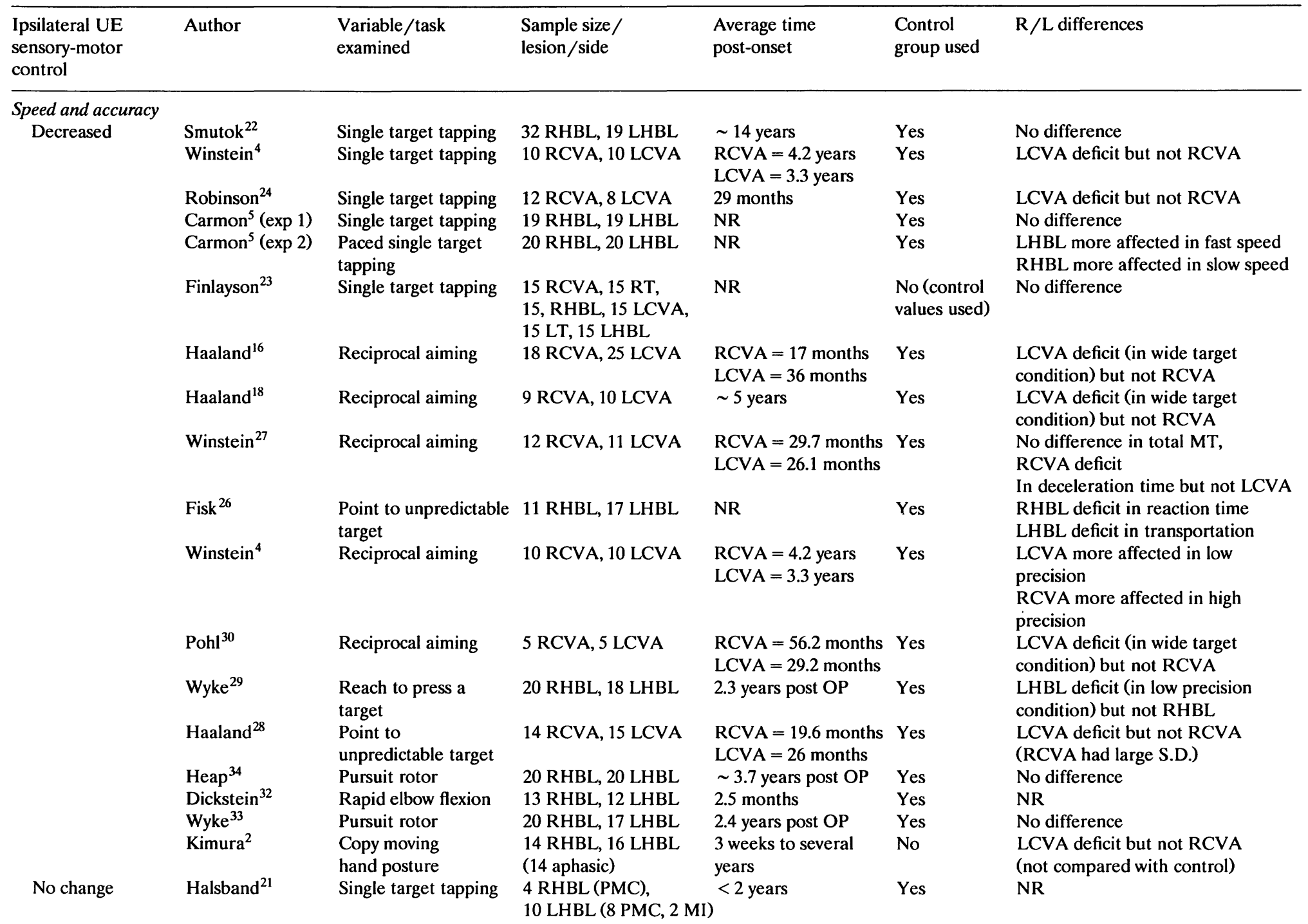


Table 1 (continued)

\begin{tabular}{|c|c|c|c|c|c|c|}
\hline $\begin{array}{l}\text { Ipsilateral UE } \\
\text { sensory-motor } \\
\text { control }\end{array}$ & Author & $\begin{array}{l}\text { Variable/task } \\
\text { examined }\end{array}$ & $\begin{array}{l}\text { Sample size/ } \\
\text { lesion/side }\end{array}$ & $\begin{array}{l}\text { Average time } \\
\text { post-onset }\end{array}$ & $\begin{array}{l}\text { Control } \\
\text { group used }\end{array}$ & $\mathrm{R} / \mathrm{L}$ differences \\
\hline \multicolumn{7}{|c|}{ Speed and accuracy } \\
\hline \multirow[t]{7}{*}{ No change } & Haaland ${ }^{3}$ & Finger tapping & 10 RCVA, 10 LCVA & $\sim 5$ years & Yes & No difference \\
\hline & Haaland $^{16}$ & Finger tapping & 18 RCVA, 25 LCVA & $\begin{array}{l}\text { RCVA }=17 \text { months } \\
\text { LCVA }=36 \text { months }\end{array}$ & Yes & No difference \\
\hline & Haaland ${ }^{18}$ & Finger tapping & 9 RCVA, 10 LCVA & $\sim 5$ years & Yes & No difference \\
\hline & Haaland $^{19}$ & Finger tapping & 17 RCVA, 26 LCVA & $\sim 25.4$ months & Yes & No difference \\
\hline & Haaland ${ }^{20}$ & Finger tapping & $\sim 150$ Brain tumor & NR & Yes & No difference \\
\hline & Kimura $^{17}$ & Finger tapping & $\begin{array}{l}16 \text { RCVA, } 29 \text { LCVA } \\
\text { (14 aphasic, } \\
15 \text { non-aphasic) }\end{array}$ & $\begin{array}{l}<1 \text { month }(20 \mathrm{Ss}) \\
>1 \text { month }(25 \mathrm{Ss})\end{array}$ & No & NA \\
\hline & Vaughan $^{15}$ & Finger tapping & 18 RHBL, 17 LHBL & NR & Yes & No difference \\
\hline \multicolumn{7}{|l|}{ Coordination } \\
\hline \multirow[t]{2}{*}{ Decreased } & Pohl ${ }^{30}$ & $\begin{array}{l}\text { Reciprocal aiming/ } \\
\text { relative timing }\end{array}$ & 5 RCVA, 5 LCVA & $\begin{array}{l}\text { RCVA }=56.2 \text { months } \\
\text { LCVA } 29.2 \text { months }\end{array}$ & Yes & $\begin{array}{l}\text { Relative movement time } \\
\text { deficit in LCVA but } \\
\text { not RCVA }\end{array}$ \\
\hline & Desrosiers $^{43}$ & Finger to nose & 14 RCVA, 29 LCVA & 25.1 months & Yes & No difference \\
\hline \multirow[t]{5}{*}{ No change } & Chleffi $^{38}$ & Reaching and grasping & 1 RCVA & 1 year & Yes & NA \\
\hline & Jeannerod $^{37}$ & Pointing at target & 1 LCVA & $\sim 7$ years & No & NA \\
\hline & Fisk $^{26}$ & $\begin{array}{l}\text { Point to unpredictable } \\
\text { target }\end{array}$ & 11 RHBL, 17 LHBL & NR & Yes & $\begin{array}{l}\text { No difference (pattern of } \\
\text { movement was similar) }\end{array}$ \\
\hline & Trombly ${ }^{39}$ & Reaching & 5 RHBL & 16 weeks to 30 weeks & No & NA \\
\hline & Trombly $^{40}$ & Reaching & 5 RHBL & 9 weeks to 22.3 weeks & No & NA \\
\hline \multicolumn{7}{|c|}{ Sensory perception } \\
\hline \multirow[t]{8}{*}{ Decreased } & Desrosiers ${ }^{43}$ & Thumb kinesthesia & 14 RCVA, 29 LCVA & 25.1 months & Yes & No difference \\
\hline & Haaland $^{28}$ & $\begin{array}{l}\text { Two-point discrimination } \\
\text { of forearm }\end{array}$ & 14 RCVA, 15 LCVA & $\begin{array}{l}\text { RCVA }=19.6 \text { months } \\
\text { LCVA }=26 \text { months }\end{array}$ & Yes & No difference \\
\hline & Haaland $^{18}$ & $\begin{array}{l}\text { Two-point discrimination } \\
\text { of forearm }\end{array}$ & 9 RCVA, 10 LCVA & $\sim 5$ years & Yes & No difference \\
\hline & Vaughan $^{15}$ & Two-point discrimination & 18 RHBL, 17 LHBL & NR & Yes & $\begin{array}{l}\text { RHBL deficit but } \\
\text { not LHBL }\end{array}$ \\
\hline & Boli $^{46}$ & Tactile perception & 30 RHBL, 30 LHBL & NR & No & $\begin{array}{l}\text { RHBL more affected } \\
\text { than LHBL }\end{array}$ \\
\hline & Carmon $^{47}$ & Tactile perception & 30 RHBL, 30 LHBL & NR & Yes & $\begin{array}{l}\text { RHBL deficit but } \\
\text { not LHBL }\end{array}$ \\
\hline & Robertson $^{52}$ & Material recognition & 10 LCVA & 24 months & Yes & NA \\
\hline & $\begin{array}{l}\text { Sartor- } \\
\text { Glittenberg }{ }^{49}\end{array}$ & Elbow kinesthesia & 13 RCVA, 7 LCVA & NR & Yes & No difference \\
\hline No change & $\mathrm{Jones}^{45}$ & Joint proprioception & 5 RHBL, 3 LHBL & 11 days & Yes & NA (too few Ss) \\
\hline
\end{tabular}


Table 1 (continued)

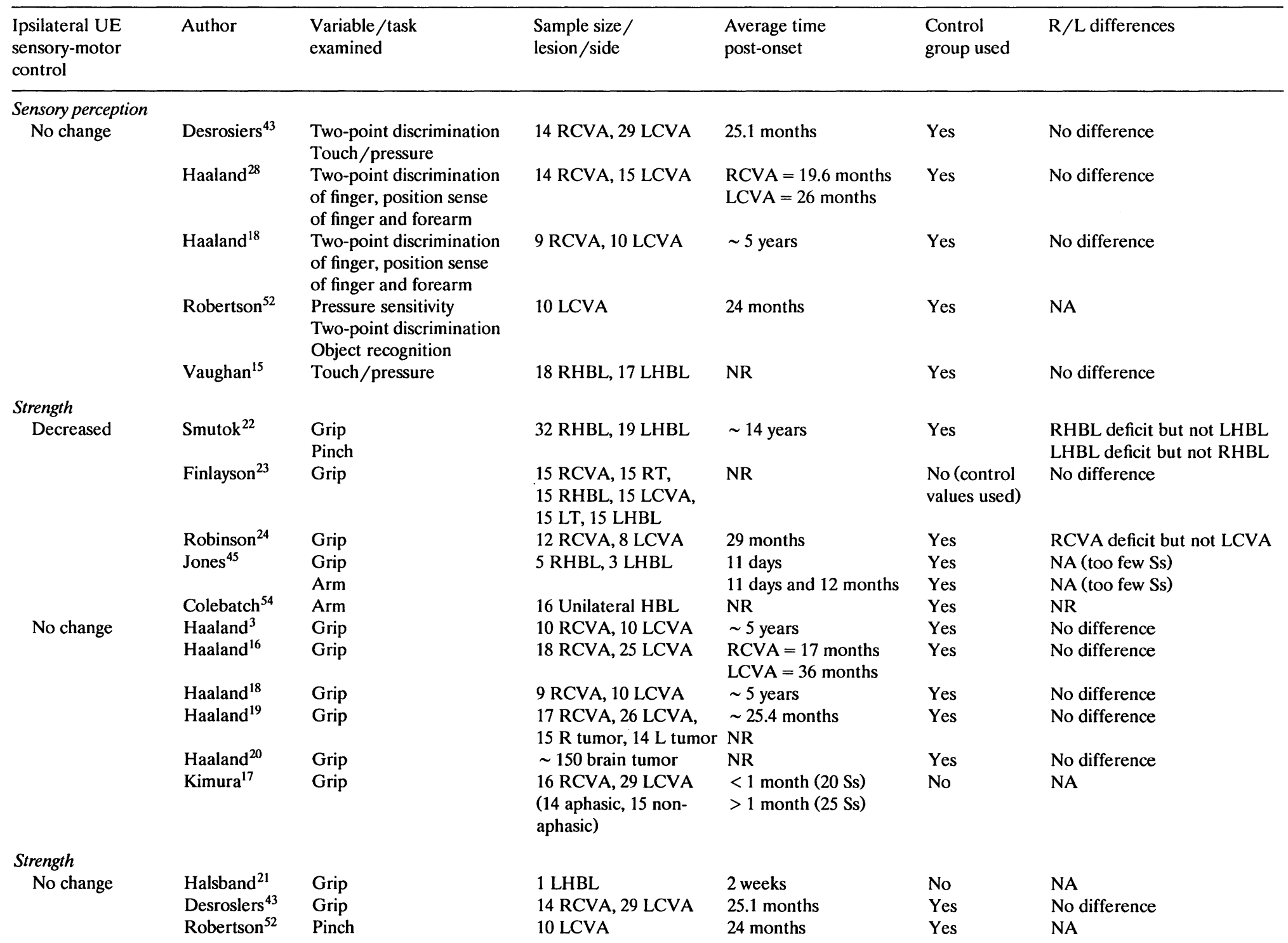


Table 1 (continued)

\begin{tabular}{|c|c|c|c|c|c|c|}
\hline $\begin{array}{l}\text { Ipsilateral UE } \\
\text { sensory-motor } \\
\text { control }\end{array}$ & Author & $\begin{array}{l}\text { Variable /task } \\
\text { examined }\end{array}$ & $\begin{array}{l}\text { Sample size/ } \\
\text { lesion/side }\end{array}$ & $\begin{array}{l}\text { Average time } \\
\text { post-onset }\end{array}$ & $\begin{array}{l}\text { Control } \\
\text { group used }\end{array}$ & $\mathrm{R} / \mathrm{L}$ differences \\
\hline \multicolumn{7}{|c|}{ Clinical assessments of UE impairment and function } \\
\hline \multirow[t]{14}{*}{ Deficit } & Robertson $^{52}$ & Jebsen test & 10 LCVA & 24 months & Yes & NA \\
\hline & Jebsen $^{60}$ & Jebsen test & 13 RCVA, 14 LCVA & NR & Yes & $\begin{array}{l}\text { RCVA deficit in } 2 \text { of } 7 \text { subtests } \\
\text { LCVA deficit in } 5 \text { of } 7 \text { subtests }\end{array}$ \\
\hline & Spauldling ${ }^{61}$ & Jebsen test & 27 RCVA, 22 LCVA & NR & Yes & $\begin{array}{l}\text { LCVA more affected than RCVA in } \\
\text { writing subtest (not compared with } \\
\text { control) }\end{array}$ \\
\hline & Desrosiers $^{43}$ & $\begin{array}{l}\text { Pegboard } \\
\text { TEMPA } \\
\text { Box and block } \\
\text { test }\end{array}$ & 14 RCVA, 29 LCVA & 25.1 months & Yes & $\begin{array}{l}\text { No difference } \\
\text { No difference (deficit showed in } 2 \text { of } 4 \\
\text { tests) } \\
\text { No difference }\end{array}$ \\
\hline & Haaland $^{18}$ & Pegboard & 9 RCVA, 10 LCVA & $\sim 5$ years & Yes & No difference \\
\hline & Haaland ${ }^{19}$ & Pegboard & 17 RCVA, 26 LCVA & $\sim 25.4$ months & Yes & No difference \\
\hline & Haaland ${ }^{20}$ & Pegboard & $\sim 150$ Brain tumor & NR & Yes & LHBL deficit but not RHBL \\
\hline & Vaughan 15 & Pegboard & 18 RHBL, 17 LHBL & NR & Yes & No difference \\
\hline & Smutok $^{22}$ & Pegboard & 32 RHBD, 19 LHBD & $\sim 14$ years & Yes & No difference \\
\hline & Tsai $^{62}$ & Pegboard & 19 RCVA, 17 LCVA & \multirow{2}{*}{\multicolumn{2}{|c|}{$\begin{array}{l}\text { RCVA }=16.9 \text { months es } \\
\text { LCVA }=19.7 \text { months }\end{array}$}} & No difference \\
\hline & & MRMT & & & LCVA $=19.7$ months & LCVA more affected than RCVA \\
\hline & & \multicolumn{2}{|l|}{ O'Connor finger } & & & (trend only) \\
\hline & & & & & & $\begin{array}{l}\text { LCVA more affected than RCVA } \\
\text { (trend only) }\end{array}$ \\
\hline & & \multicolumn{2}{|l|}{$\begin{array}{l}\text { O'Connor tweezer } \\
\text { dexterity }\end{array}$} & & & $\begin{array}{l}\text { LCVA more affected than RCVA } \\
\text { (trend only) }\end{array}$ \\
\hline No deficit & Halsband ${ }^{21}$ & Pegboard & $\begin{array}{l}4 \text { RHBL (PMC), } 10 \\
\text { LHBL (8 PMC, } 2 \text { MI) }\end{array}$ & $<2$ years & Yes & NR \\
\hline
\end{tabular}

Abbreviations: RCVA, right cerebrovascular accident; LCVA, left cerebrovascular accident; RHBL, right hemisphere brain lesion; LHBL, left hemisphere brain lesion; RT, right traumatic brain injury; LT, left traumatic brain injury; R tumor, right tumor; L tumor, left tumor; MT, movement time; OP, operation; Ss, subjects; S.D. Standard Deviation; TEMPA, Upper Extremity Performance Evaluation Test for the Elderly; MRMT, Minnesota Rate of Manipulation Test; NR, not reported; NA, not applicable. 